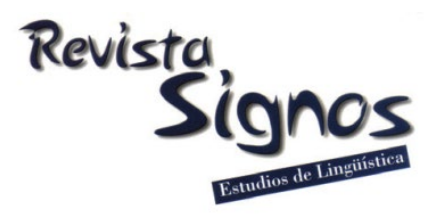

\title{
Lo concreto y lo abstracto en nominalizaciones deadjetivales
}

\section{The concrete and the abstract in deadjectival nominalizations}

\author{
Ana Clara Polakof \\ UNIVERSIDAD DE LA REPÚBLICA/ SISTEMA NACIONAL DE INVESTIGADORES \\ URUGUAY \\ yongyanzheng@fudan.edu.cn
}

Recibido: 04-IV-2018 / Aceptado: 28-XI-2018

DOI: $10.4067 /$ S0718-09342019000200410

\section{Resumen}

Este artículo analiza el comportamiento de las nominalizaciones a partir de adjetivos intersectivos no evaluativos. Primero, presenta un análisis cuantitativo que compara el uso de las nominalizaciones al de los adjetivos a partir de los cuales se forman. Segundo, hace un análisis cualitativo de las lecturas abstractas y las concretas que presentan. Defiende que, si bien nominalizaciones como 'blancura' pueden tener un significado abstracto intrínseco, presentan lecturas concretas que deben ser analizadas. Defiende, además, que la diferencia entre la lectura concreta y la abstracta radica en la estructura sintáctica del sintagma determinante que las nominalizaciones forman. Esto permite establecer que no es necesario proponer la existencia de un ítem léxico con lectura concreta y con lectura abstracta, sino que es posible asociar esa diferencia semántica a una diferencia sintáctica estructural.

Palabras Clave: Adjetivos intersectivos no evaluativos, nominalizaciones deadjetivales, abstracto, concreto, sintagma determinante. 


\begin{abstract}
This article analyses the behavior of nominalizations from non evaluative interesective adjectives. First, it presents a quantitative analysis that compares the use of these nominalizations to the one of the adjectives from which they are formed. Second, it makes a qualitative analysis of abstract and concrete uses which they present. It defends that, even if nominalizations as 'blancura' ('whiteness') have an intrinsic abstract meaning, they may present concrete readings which need to be analyzed. It further defends that it is possible to establish the difference between the concrete reading and the abstract one by analyzing the syntactic structure of the determinant phrase (DP). This allows us to establish that it is not necessary to propose the existence of a lexical item with a concrete reading and one with an abstract one. We may propose that the semantic difference is due to a difference in the syntactic structure of the DP.
\end{abstract}

Key Words: Intersective non evaluative adjectives, deadjectival nominalizations, abstract, concrete, determinant phrase.

\title{
INTRODUCCIÓN
}

Este trabajo surge a partir de nuestro interés en definir si es posible referir a lo abstracto (Polakof, 2017). Analizar dicha posibilidad nos llevó a centrarnos en el estudio de nominalizaciones a partir de adjetivos intersectivos no evaluativos, como 'blanco' o 'redondo', dentro del sintagma determinante (SD) -determinado por el artículo definido- en la posición de sujeto. Habíamos partido de la hipótesis de que dichas nominalizaciones se relacionaban inequívocamente con lo abstracto - entendido ontológicamente como aquello que no está en el espacio-tiempo y no presenta relaciones de causalidad (ser mujer es una propiedad que no está en el espacio-tiempo y que no se relaciona causalmente con nada, por ejemplo) - y, por lo tanto, debían poder ser usadas para referir a ello. Sin embargo, una vez que analizamos los datos pudimos constatar que dichas nominalizaciones tenían, además del uso que nos permite relacionarlas con lo abstracto, un uso que nos permite relacionarlas con lo concreto -entendido ontológicamente como aquello que está en el espacio-tiempo y presenta relaciones de causalidad (una explosión puede estar localizada espaciotemporalmente y causar la rotura de un edificio, por ejemplo) - y que dicho uso era más frecuente de lo esperado. A partir de este descubrimiento, nos preguntamos qué es lo que permite que las mismas (o aparentemente iguales) estructuras se relacionen con lo abstracto y con lo concreto. Esa es la pregunta que este trabajo busca responder.

Las nominalizaciones deajdetivales han sido poco estudiadas en el marco generativo en comparación con, por ejemplo, las deverbales. Su estudio es relativamente reciente. Suelen asociarse con su sentido abstracto (término que no suele estar bien definido), en casos como 'la blancura es una propiedad' (ver McNally \& de Swart, 2011). Sin embargo, como Alexiadou (2013) ha notado, estas nominalizaciones pueden tener un comportamiento tipo eventivo (en algunos casos 
será estativo), que puede verse en 'la blancura de la ropa duró 10 segundos'. Nosotros defendemos que este tipo de usos está relacionado con lo concreto: 'la blancura de la ropa' en la oración anterior nos permite localizar espacio-temporalmente la blancura en la ropa y esa blancura de la ropa, un estado determinado, puede haber sido causada por el lavado de la ropa, un evento determinado (ver Polakof, 2017, Polakof en prensa). Además de poseer estas lecturas, dichas nominalizaciones pueden tener lecturas concretas de cosas, como en 'la blancura esa en la pared es una mancha de humedad'. En este caso, podemos entender que se relacionan con un objeto en el mundo, una mancha de humedad.

$\mathrm{El}$ hecho de que estas nominalizaciones puedan alternar entre una lectura abstracta y una lectura concreta que se relaciona con entidades abstractas y con entidades concretas en el mundo ha sido poco estudiado. En este trabajo, nos proponemos mostrar cuáles son los factores sintácticos y semánticos que ayudan a la existencia de esta ambigüedad interpretativa. Para esto, restringimos el trabajo a las nominalizaciones deadjetivales intersectivas no evaluativas, y usamos el CORPES de la RAE para analizarlas y compararlas con el uso de los adjetivos base, para ver la preferencia de los hablantes respecto del uso de los adjetivos contra el de sus respectivas nominalizaciones. Finalmente, argumentamos que las diferencias entre ambas lecturas se deben a la estructura sintáctica del SD y no al contenido léxico de las nominalizaciones.

\section{Marco teórico}

Nos aproximamos a la nominalización a partir del planteo de Chierchia (1982: 305). Se extiende, por lo tanto, la terminología lingüística tradicional para dar cuenta de todos los "syntactic and semantic processes by which sentences, verb phrases, common nouns and other items are 'transformed' into noun phrases (...)". Se puede entender, entonces, que la nominalización que concierne los adjetivos es toda aquella que permite que un adjetivo en posición predicativa sea transformado en un complemento del determinante, pudiendo ocupar posiciones argumentales. Estas nominalizaciones han sido poco estudiadas. Aquellas que se forman a partir de adjetivos evaluativos (como 'honestidad', 'generosidad', etc.) han sido objeto de la mayoría de los análisis sobre nominalizaciones deadjetivales ${ }^{1}$ (Alexiadou, 2011; Martin, 2010; Roy, 2010; Fábregas, 2011; Villalba, 2013; entre otros), pues parecen tener un uso más frecuente. Las nominalizaciones a partir de adjetivos calificativos intersectivos no evaluativos, en las que este trabajo se centra, han sido menos estudiadas. Hay, sin embargo, estudios (Alexiadou, 2013; Kennedy \& McNally, 2010; McNally \& de Swart, 2011; McNally \& de Swart, 2015) que se han enfocado en nominalizaciones a partir de colores que pueden ser adjetivos calificativos intersectivos (ver Hansen \& Chemla 2017, por usos no intersectivos de los adjetivos de color). 
McNally y de Swart (2011) reconocen tres tipos de formas relacionadas con el color en holandés: una forma no flexionada (uninflected), una nominalización deadjetival (adjectival nominalizations), y una flexionada (inflected). La forma no flexionada es nominal, denota la descripción del color y se combina con el artículo neutro (bet rood van de aardbeien/ 'el rojo de las frutillas'). La forma equivalente del español también describe el color. La nominalización deadjetival es nominal, no neutra y se construye a partir del sufijo -heid (De roodheid van de huid/ 'La rojez de la piel'). En español, dicha nominalización se puede formar con distintos sufijos, como -ura, -or, -ez, entre otros ('la blancura de la pared'). La forma flexionada del holandés no es fácil de caracterizar. Según las McNally y de Swart (2011), ha sido difícil establecer si debe ser analizada como un proceso que cambia la categoría del adjetivo a nombre o como un adjetivo con un nominal elidido (Het rode van de aardbeien/ 'El rojo [aspecto] de las frutillas'). Este último tipo es, de acuerdo con las autoras, parecido a las formas de color que seleccionan el artículo neutro en español ('lo rojo de las frutillas'). Respecto de la semántica de estas formas, proponen que el primer tipo se refiere al tono de color de las cosas que tienen ese color determinado (a un subtipo), o pueden también denotar la realización de aquel tono en el objeto); que el segundo tipo puede tener un uso proxy que indica una característica determinada (por ejemplo, la luz roja del semáforo indica que tenemos que parar) o puede denotar el conjunto del subtipo denotado por el color); y que, en el último caso, el adjetivo continúa siendo un adjetivo, o sea, no hubo una transformación en la categoría del adjetivo (la interpretación formal puede verse en McNally \& DeSwart, 2011). Las autoras defienden, también, que los primeros dos tipos analizados son nombres: las nominalizaciones deadjetivales pueden ser pluralizadas ('las blancuras de la casa'), los dos pueden ser modificados por adjetivos ('el lindo rojo de la flor', 'la perfecta blancura de la cara'), y pueden combinarse con varios determinantes ('ese rojo', 'esa blancura') (McNally \& DeSwart, 2011).

Un problema que ese trabajo presenta es que McNally y DeSwart (2011) parecen defender que la forma no flexionada puede referir a tipos/especies y a sus realizaciones, pero que las nominalizaciones deadjetivales se relacionan solo con tipos/especies (o con el uso proxy). Sin embargo, Alexiadou (2013) nota, con base en Martin (2010), que las nominalizaciones deadjetivales de color pueden combinarse con predicados de duración y estar restringidas en el espacio-tiempo, como los ejemplos a continuación. O sea, pueden interpretarse como eventualidades y pueden, por lo tanto, tener lecturas concretas además de las abstractas. Alexiadou (2013) da ejemplos del griego: $i$ asprila kratise deka meres / 'la blancura duró 10 dias' e $i$ kokinila sto heri mu me anisibise/'la rojez de mi mano me preocupaba'. Estos ejemplos son trasladables al español (como puede observarse en las traducciones), en donde parece clara la lectura concreta de la nominalización. Otro problema, relacionado con la falta de explicitación de algunas cuestiones en dicho artículo, es que las autoras hablan de la combinación de las nominalizaciones con adjetivos, demostrativos y de la posibilidad de pluralización, sin analizar cómo afecta dicho cambio la lectura de esas 
nominalizaciones. Como mostraremos al analizarlas en la posición de sujeto, la pluralización puede transformar una lectura abstracta en una concreta, y lo mismo puede suceder con la combinación de esas nominalizaciones con demostrativos y adjetivos.

\section{Marco metodológico}

Decidimos hacer nuestra investigación con nominalizaciones a partir de adjetivos calificativos intersectivos, porque, con ellos, es posible evitar la relatividad interpretativa que surge a partir del análisis de los subsectivos. Descartamos, también, los adjetivos evaluativos porque dependen de cómo el ser humano caracteriza un individuo. Como queremos centrarnos en significados abstractos que sean independientes de nuestras conceptualizaciones, los evitamos. Son estos significados los que pueden relacionarse más claramente con el dominio de lo abstracto (aquel en el que las entidades no están en el espacio-tiempo y no presentan relaciones de causalidad) en la realidad. Hicimos la investigación a partir del trabajo con corpus, pues nos permite establecer patrones de comportamiento de las nominalizaciones de manera objetiva.

\subsection{Corpus}

Utilizamos el corpus CORPES de la Real Academia Española (RAE) para nuestro trabajo porque nos permite una búsqueda centrada en los datos. El CORPES es un recurso en construcción que contiene documentos de distintas variedades del español pertenecientes al siglo XXI. Presenta distintos filtros que nos permiten crear un subcorpus que se atenga a las características que buscamos. El único filtro que utilizamos fue el que nos permite eliminar los documentos ficcionales. Ellos fueron eliminados para evitar usos demasiado metafóricos y poco comunes (como 'el mordisco de la muerte le había mostrado la blancura de su ferocidad' [CORPES XXI]). Utilizamos la versión 0.83 (1 de junio de 2016), en la cual aproximadamente el $30 \%$ de los documentos pertenece a España y el $70 \%$ a América. Las formas contenidas en ese subcorpus llegan casi a los 158 millones (CORPES, 2016, Datos generales), y nuestras búsquedas fueron hechas desde agosto de 2016 hasta enero de 2017.

\subsection{Procedimiento}

\subsubsection{Procedimiento del análisis cuantitativo}

Buscamos en el CORPES ciertos adjetivos y sus nominalizaciones. Los adjetivos buscados fueron 'rojo', 'blanco', ‘negro', 'verde', azul', 'redondo' y 'alto ${ }^{2}$. La búsqueda fue hecha utilizando el 'comodín'. Esto nos permitió sustituir la vocal final del adjetivo (en los casos en los que hay variación de género) por el símbolo de interrogación (?) que hace que la búsqueda en el CORPES dé como resultado final las formas de los 
adjetivos en los dos géneros; busca tanto las apariciones de 'blanco' como de 'blanca'. Hicimos la búsqueda de esa manera para obtener datos representativos. Los adjetivos fueron buscados solo en el singular, porque asumimos que las nominalizaciones que íbamos a analizar se formarían a partir de oraciones en las que predicarían algo de un sujeto en singular. Esta hipótesis estaba equivocada, ya que encontramos nominalizaciones como 'la blancura de las casas'. Sin embargo, como nuestro interés era analizar las nominalizaciones, no volvimos a realizar la búsqueda incluyendo los adjetivos en plural. Después, buscamos registros de nominalizaciones a partir de esos adjetivos en singular. En el caso de los adjetivos de color, analizamos dos formas de nominalización: la no sufijada y la nominalización deadjetival. ${ }^{3}$ Todos presentan la forma nominal no sufijada, pero no todos los adjetivos de color forman nominalizaciones deadjetivales. En el caso de los otros adjetivos, solo se registra la versión sufijada, porque la otra tiene una lectura humana, la llamada Human Construction (McNally \& DeSwart, 2015), que presenta un comportamiento que difiere del que queremos analizar. Son casos como 'Los ricos solo se hacen más ricos', donde se presenta un adjetivo que describe una característica determinada de los humanos y que está, aparentemente, nominalizado. Son analizados por McNally y de Swart (2015: 323) como adjetivos que modifican un nombre nulo que "contributes a free variable and the sortal restriction that the value of this variable must be human". O sea, no son nombres. Por lo tanto, no fueron considerados en nuestra investigación que se centra en el estudio de la polisemia abstracto/concreto en nombres y no en adjetivos, y -aunque fueran nombres- presentan una lectura humana que no interesa al analizar lo abstracto (en el sentido aquí utilizado).

\subsubsection{Procedimiento del análisis cualitativo}

El análisis cualitativo fue hecho con un número restringido de registros, pues no todas las construcciones aparecen con frecuencia suficiente para generar datos cualitativamente diferentes ('negrura', por ejemplo, presenta solo 13 registros) y, además, el hecho de que haya muchas apariciones no significa que sean todas cualitativamente significativas. Por ejemplo, si el mismo sintagma determinante (SD) con los mismos complementos- aparece reiterado en la misma posición sintáctica, ese no es un dato cualitativamente significativo (aunque podría ser cuantitativamente significativo). Debido a esas cuestiones, decidimos formar un subcorpus del siguiente modo. Seleccionamos 10 registros de cada una de las nominalizaciones aquí analizadas y nos aseguramos de que seleccionaran complementos diferentes, para evitar duplicar el análisis. Por ejemplo, 'la negrura de la noche' fue registrada varias veces, pero solo dos registros de ese SD fueron incluidos en el subcorpus porque presentan una estructura sintáctica diferente ('la negrura de la noche y de la niebla' contra 'la negrura de la noche'). Por eso, en el caso de 'la negrura de' + SD hay solo 9 registros, pues de los 13 , cinco eran equivalentes a 'la negrura de la noche'. Intentamos, cuando fue 
posible, seleccionar SD que apareciesen en posiciones sintácticas diferentes: sujeto, objeto directo, complemento preposicional, entre otros. ${ }^{4}$

Después de haber registrado estos usos, decidimos trabajar con estas estructuras en la posición de sujeto, porque este es un contexto referencialmente fuerte -en el que se supone que es posible relacionar el SD con la realidad- que nos permite enfocarnos en la lectura abstracta/concreta de las nominalizaciones. Al analizar una única posición sintáctica, podemos estudiar este comportamiento sin cuestionarnos si la interpretación varía según la posición sintáctica que el SD ocupa. Un claro ejemplo de esto sería un SD que al estar en la posición de sujeto fuese fuertemente referencial (con una entidad concreta con la cual claramente se relaciona), como en 'El hospital es ese edificio de ahí' en donde se asume que hay un objeto con el que 'el hospital' se corresponde, pero que tuviese una lectura incorporada cuando está en la posición interna (ver Klein, Gegg-Harrison, Carlson \& Tanenhaus, 2013), como en 'Ana fue al hospital' en donde se asume que Ana, además de ir al hospital, fue al médico, a hacerse exámenes, etc.

\section{Resultados}

\subsection{Análisis cuantitativo de las nominalizaciones}

La tabla que se presenta a continuación muestra los datos obtenidos. En la primera columna, presentamos la forma base del adjetivo. En los casos en que el adjetivo presenta variación morfofonológicamente relevante de género, la base se presenta con el signo '?', y en los que no lo hace, no. En la segunda, se presenta el número de registros del adjetivo en el corpus. En la tercera, la forma del SD que contiene la nominalización que no presenta un sufijo fonológicamente expresado. En la cuarta, el número de registros de esos SDs. En quinto lugar, la forma del SD que contiene la nominalización con sufijo fonológicamente expresado. En último lugar, el número de registros de esos SDs.

Tabla 1. Registros de adjetivos y SDs con nominalizaciones deadjetivales.

\begin{tabular}{|c|c|c|c|c|c|c|}
\hline & Adjetivo & $\mathbf{N}^{\circ}$ & Artículo + nombre sin sufijo & $\mathbf{N}^{\circ}$ & Artículo + nominalización sufijada & $\mathbf{N}^{\circ}$ \\
\hline (1) & Azul & 6456 & $\mathrm{El}$ azul de $+\mathrm{SD}$ & 80 & --------------- & 0 \\
\hline (2) & blanc? & 24086 & El blanco de + SD & 62 & La blancura de + SD & 27 \\
\hline (3) & Negr? & 17865 & El negro de + SD & 35 & La negrura de + SD & 13 \\
\hline (4) & Roj? & 11815 & El rojo de + SD & 56 & -------------- & 0 \\
\hline (5) & Verde & 8941 & El verde de + SD & 77 & $\mathrm{El}$ verdor de $+\mathrm{SD}^{5}$ & 29 \\
\hline (6) & Alt? & 55966 & - - & 0 & $\mathrm{La}$ altura de $+\mathrm{SD}$ & 597 \\
\hline (7) & Redond? & 1975 & -------------- & 0 & La redondez de + SD & 28 \\
\hline
\end{tabular}

Esos números pueden no ser cuantitativamente significativos, porque son bajos. Sin embargo, la comparación numérica de las nominalizaciones contra la de los adjetivos sí lo es. Nos permite establecer que, si tomamos como 100\% el valor de apariciones de los adjetivos en el corpus y comprobamos - con relación a ese $100 \%$ 
cuál es el porcentaje de uso de las nominalizaciones, ninguna nominalización supera el $2 \%$ del uso del adjetivo. O sea, se utiliza la forma adjetiva más de un $98 \%$ más que la forma nominal para describir cosas y sus propiedades en el mundo. Esos datos podrían llevarnos a concluir, entre otras posibles conclusiones (como que la asimetría se deba a una diferencia importante en la carga computacional de estos ítems), que estas nominalizaciones no son usadas frecuentemente por hablantes del español para denotar las propiedades abstractas y las cosas que ellas complementan. Es decir, para adscribir propiedades a un individuo determinado, preferimos utilizar el adjetivo predicando al nombre y no su nominalización.

El nombre 'altura', en el sintagma determinante, debe ser analizado aquí, pues la base adjetiva puede ser clasificada como subsectiva o como intersectiva. El uso del adjetivo 'alto' como subsectivo es relativo al nombre que está modificando. Por ejemplo, que una persona sea 'alta' puede depender del subconjunto en el cual el ser 'alto' sea evaluado. Sería posible afirmar algo como 'Él es alto para jugador de fútbol, pero bajo para jugador de básquetbol' (autogenerado), ejemplo que permite mostrar que en este uso el adjetivo es relativo. Sin embargo, el adjetivo 'alto' tiene otro significado menos usado, a partir del cual podemos definir que se forma 'altura'. Ese significado puede ser encontrado en el Diccionario de la Lengua Española (DLE), en la entrada: 'alto1. 1. levantado, elevado sobre la tierra'. Es posible considerar, entonces, que el nombre fue derivado a partir del uso intersectivo. La altura de un objeto $\mathrm{x}$ es una distancia determinada a partir de un punto tomado como referencia. Si bien puede afirmarse que esa fijación es relativa, todas las cosas que tienen una 'altura $x$ ' tienen esa altura $\mathrm{x}$ sin importar con quiénes se comparen, y se forma a partir del significado intersectivo (y no del subsectivo) del adjetivo ${ }^{6}$.

Finalizamos esta sección estableciendo que, en general, se utiliza el adjetivo para calificar una cosa y que la nominalización (en su respectivo SD) que nos permitiría denotar lo abstracto (en el sentido utilizado aquí: aquello que no está en el espaciotiempo y no presenta relaciones de causalidad), en principio, tiene una baja frecuencia de uso (en comparación con el uso del adjetivo como modificador del nombre). No podemos, aún, afirmar si la afiliación léxica (nombres contra adjetivos) está directamente relacionada con las lecturas abstractas. Sin embargo, parece ser una hipótesis viable para perseguir en el futuro. Para establecer si estas nominalizaciones abstractas poseen también una lectura concreta debemos hacer un análisis cualitativo que nos permita establecer los usos de este tipo de nominalizaciones (por más que no sean demasiado usadas).

\subsection{Análisis cualitativo de las nominalizaciones}

Las nominalizaciones analizadas aparecen como sujeto de predicados copulativos (y uno con un verbo pseudocopulativo): 'altura', 'blanco' y 'verdor'; y como sujeto de predicados no copulativos: 'altura', 'azul', 'blancura', 'negro', 'negrura', 'rojo', 'verde' y 
'verdor'. Que puedan aparecer como sujeto de cualquier tipo de predicado nos permite suponer que funcionan como cualquier otro sustantivo. Deben poder tener un uso concreto además del abstracto para funcionar como sujeto de algunas oraciones predicativas. O sea, las nominalizaciones tienen dos posibles lecturas. Esto parece concordar inicialmente con el análisis propuesto por McNally y DeSwart (2011) para nombres de color no sufijados, aunque para el caso de las nominalizaciones deadjetivales -que las autoras relacionan solo con lo abstracto- debemos adicionar la propuesta de Alexiadou (2013) que reconoce que, además de tipos/especies abstractos, esas nominalizaciones identifican eventualidades que podemos relacionar con entidades concretas, pues pueden estar espacio-temporalmente localizadas.

Presentamos, primero, las oraciones copulativas (primero las propiamente copulativas y segundo la pseudocopulativa), y, segundo, las predicativas. Ambas con los sintagmas en la posición de sujeto:

(8) La altura de este cerro es de 4872 m sobre el nivel del mar (...)

(9) (...) quizá sea el verdor de los bosques mediterráneos lo más especial y exclusivo

(10) el blanco de la nieve parece que intenta iluminar un poco la oscuridad del invierno.

(11) Estas características de las nominalizaciones deadjetivales también fueron notadas por Villalba (2013) para casos como 'belleza', a partir de adjetivos evaluativos. Estas características de las nominalizaciones deadjetivales también fueron notadas por Villalba (2013) para casos como 'belleza', a partir de adjetivos evaluativos. Estas características de las nominalizaciones deadjetivales también fueron notadas por Villalba (2013) para casos como 'belleza', a partir de adjetivos evaluativos.

(12) (...) el azul de sus cuadros se aclara a medida que se avanza del Salón de la Guerra al Salón de la Paz.

(13) (...) la blancura de las casas del centro del país constituía una especie de legado colonial.

(14) Los grises del fondo, el azul del cuello y el negro de la sotana, demuestran una voluntad de estilo que se inscribe en el concepto de la pintura-pintura (...)

(15) (...) la negrura del fuel lanzado a sus costas tras el accidente del Prestige les provoca una valoración del futuro del mismo color.

(16) (...) la redondez de su rostro había desaparecido (...)

(17) El azul del cielo y el rojo de los atardeceres se deben a la dispersión de la luz $\operatorname{solar}(\ldots)$ 
(18) El amarillo de los muebles y el rojo de las baldosas ponen notas alegres y luminosas.

(19) El rojo de la sangre lo invade todo.

(20) (...) el rojo brillante de la cubierta se impone sobre el resto del espacio

(21) El verde de las vides sirve de marco perfecto para llegar a los muros blancos de Le Provençal (...)

(22) (...) el blanco de su vestido hace juego con su mirada que es transparente.

(23) (...) el verde de las primeras hojas anuncia el triunfo de la vida (...)

(24) (...) sobresale el esplendor de los tomates o el verdor de los pimientos.

(25) El verdor de la montaña lo penetra todo.

Ninguna de nuestras oraciones presenta el sujeto paradigmático, pues ninguno de los sujetos "refers to a well-defined, non-abstract object (...)" (Higgins, 1979: 224). Esta característica es compartida por las oraciones de 8 a 25, que se diferencian, en primera instancia, según su carácter copulativo o predicativo. La literatura establece que esos dos tipos de oraciones tienen un comportamiento diferente y, por ese motivo, analizamos, en primer lugar, las copulativas (8 y 9), y en segundo lugar, las predicativas no copulativas (11-25).

Las oraciones copulativas se dividen, normalmente, en dos tipos: uno en el cual los dos sintagmas son referenciales, y otro donde el sintagma que aparece en la posición predicativa no es referencial (Leborans, 1999). Si consideramos solo esa clasificación, es posible establecer que las dos oraciones analizadas aquí son del segundo tipo ${ }^{7}$. No hay, en la posición predicativa, estructuras que puedan ser fuertemente referenciales: en 8 ('La altura de este cerro es de $4872 \mathrm{~m}$ sobre el nivel del mar'), tenemos un complemento preposicional y, en 9 ('quizá sea el verdor de los bosques mediterráneos lo más especial y exclusivo'), un sintagma determinado por el artículo neutro - no referencial - 'lo's. La oración 8 involucra un SD cuantitativo complementado por un sintagma preposicional ('de $4872 \mathrm{~m}$ sobre el nivel del mar') pos-copular. En esa oración, entonces, el verbo copulativo une el sintagma preposicional al SD para formar una oración copulativa predicativa. La oración 9 puede ser clasificada como copulativa especificativa, pues se especifica con ella el sujeto. Ella tiene la característica de presentar el SD que generalmente aparece en posición pre-copular ('lo más especial y exclusivo') pospuesto al sujeto profundo de la especificativa ('el verdor de los bosques mediterráneos') (ver nota 35 en Leborans, 1991). Es posible notar que, en esta oración, se pierde el foco (posición no paradigmática del sujeto que le da más importancia a la información contenida en el SD analizado en esta oración) del sujeto profundo. La combinación de estos dos sujetos de oraciones copulativas con 
predicados no referenciales parece llevarnos a una lectura no referencial y abstracta de los sujetos. Sin embargo, para entender su relación con lo abstracto, debemos esperar al análisis hecho para las oraciones estativas (no copulativas) que incluyen nominalizaciones que denotan lo abstracto.

La oración 10 ('el blanco de la nieve parece que intenta iluminar un poco la oscuridad del invierno') incluye el verbo pseudocopulativo 'parecer'. Es pseudocopulativo pues presenta el predicado relacionado con la apariencia del sujeto (Morimoto \& Pavón Lucero, 2006: 2). Es un verbo no asertivo, pues no hay compromiso con la verdad de lo que es dicho (es no factivo). Sin embargo, en esa oración, es complementado por una subordinada que parece formar con él una semiperífrasis (RAE-ASALE, 2010, la sección 28.1.3). O sea, 'parecer' no se comporta como verbo pleno, y el significado que impera en la construcción es el de 'iluminar'. Podemos, entonces, afirmar que la persona que usó esa oración no se compromete con la blancura de la nieve ni con la iluminación de la oscuridad. El verbo 'parecer' aparece como modalizador de la oración y el significado de iluminar prima sobre el suyo, lo que aproxima el ejemplo 10 a los siguientes casos de predicados no copulativos, y parece cargar el sintagma con significado concreto.

Para analizar las oraciones no copulativas (11-25), usamos la noción de aspecto léxico, las clasificamos y analizamos según el aspecto que en ellas se presente, y después vemos si este influye en la interpretación del sujeto o no. ${ }^{9}$ Vendler (1967) fue uno de los precursores en la discusión sobre aspecto léxico. ${ }^{10}$ Él propone que las oraciones pueden ser clasificadas como actividades, realizaciones, logros o estados. Las actividades presentan la acción como continua y no interrumpida. Verbos como 'correr' y 'nadar' son prototípicamente actividades. Las realizaciones involucran un proceso que es continuo y termina. Predicados como 'leer un libro', o 'tomar un helado' son prototípicamente realizaciones. Los logros son acciones puntuales que no tienen duración. Ejemplos clásicos son 'llegar a la cima', 'ganar una carrera', entre otros. Finalmente, los estados perduran en el tiempo y, diferente de las actividades, no son dinámicos. Normalmente, los ejemplos dados de verbos de estado son 'amar', 'conocer', entre otros. Como podrá haber sido observado, el aspecto léxico no depende solo del verbo, sino también de los argumentos que recibe. O sea, mismo siendo 'correr' un verbo prototípicamente de actividad, es posible modificarlo y transformarlo en una realización, como en 'correr una carrera'. De esas clases de predicados, las actividades y las realizaciones son generalmente acciones, mientras que los estados y algunos logros pueden no ser acciones (Vendler, 1967).

Hay algunos tests lingüísticos que permiten la clasificación de las oraciones en actividades, estados, logros o realizaciones. Los estados no pueden combinarse con tiempos progresivos, pero las actividades, realizaciones y logros sí ('*Estoy siendo mujer', contra 'estoy corriendo', 'estoy leyendo un libro', 'estoy llegando'). Los verbos de actividad y realización pueden formar perífrasis con 'terminar de', y los de logro y 
de estado no ('Terminé de correr' y 'terminé de leer el libro', contra ‘*terminé de ser mujer', '*terminé de llegar'). Las realizaciones y los logros son delimitados, pero las actividades y los estados no ('Leí el libro en dos horas', contra '*Fui mujer en dos horas', '*corrí en dos horas'). Los verbos de estado y, a veces, las realizaciones, pueden combinarse con durante x, pero los logros no ('Viví en Uruguay durante 20 años', 'corrí durante dos horas', 'leí el libro durante dos horas', contra '*Llegué durante dos horas'). Ahora, no hay un test que sea suficiente por sí mismo. Como Arche (2016) menciona, la combinación con 'durante $\mathrm{x}$ ', por ejemplo, no siempre es útil para diferenciar eventos delimitados de no delimitados, pues, como vimos, puede combinarse con algunos verbos de realización. Entonces, si aplicamos cuidadosamente esos tests a las oraciones registradas, obtenemos lo siguiente:

Tabla 2. Aspecto Léxico de las oraciones analizadas.

\begin{tabular}{|c|c|c|c|}
\hline Oración $\mathrm{n}^{\circ}$ & Duración & Delimitación & Dinamicidad \\
\hline (11) & Sí & Sí & Sí \\
\hline (12) & Sí & Sí & Sí \\
\hline (13) & Sí & No & No \\
\hline (14) & Sí & No & No \\
\hline (15) & Sí & Sí & Sí \\
\hline (16) & Sí & Sí & Sí \\
\hline (17) & Sí & ¿No o Sí? & ¿No o Sí? \\
\hline (18) & Sí & No & No \\
\hline (19) & Sí & Sí & Sí \\
\hline (20) & Sí & $\mathrm{No}$ & No \\
\hline (21) & Sí & No & No \\
\hline (22) & Sí & No & No \\
\hline (23) & Sí & ¿Sí o No? & ¿Sí o No? \\
\hline (24) & Sí & No & No \\
\hline (25) & Sí & Sí & Sí \\
\hline
\end{tabular}

Algunas de las clasificaciones son difíciles de hacer, pues algunas lecturas parecen depender de una cierta metaforización por parte del usuario de la lengua. De todas maneras, fue posible establecer que las oraciones pueden ser clasificadas como estados y como realizaciones. No hay logros ni actividades. Los logros parecen involucrar alguna causalidad simple que no se presenta en nuestros ejemplos. Las actividades parecen involucrar procesos 'simples', y parecería ser que las nominalizaciones que involucran eventualidades (presentan estructura argumental, temporalidad y -como otras nominalizaciones deadjetivales con lecturas de eventualidad- no pueden ser pluralizadas, Villalba, 2013) serían evitadas como sujetos de actividades, pues involucrarían el aumento de la complejidad del proceso. Parece, por lo tanto, más natural que, en los casos en los que las nominalizaciones aparecen como sujeto de predicados de proceso, sean sujeto de realizaciones que son procesos complejos.

Analizamos, en primer lugar, las realizaciones y, en segundo lugar, los estados. Como ya establecimos que estas nominalizaciones pueden tener lecturas concretas, queremos ver si al aparecer en realizaciones (que involucran causalidad) presentan solo 
una lectura concreta y si en los estados (que no parecen involucrar causalidad) presentan solo una lectura abstracta. Para analizar qué es lo que cambia la interpretación de abstracto a concreto, debemos analizar las realizaciones y los estados.

Los sintagmas que son sujeto de realizaciones involucran, mayormente, nominalizaciones sufijadas. Son excepciones casos como 'rojo' y 'azul' que no presentan versiones sufijadas en el corpus. Por lo tanto, podemos establecer que son las nominalizaciones sufijadas las que son escogidas, contra los nombres de color, cuando estamos frente a oraciones que involucran procesos. Sin embargo, en los casos en los que no existe una versión sufijada, el nombre de color cumple dicho rol. O sea, si tenemos dos variantes, se prefiere la sufijada como sujeto de proceso, pero si la única opción es la no sufijada se usa dicha forma. Las lecturas de realización parecen depender del punto de vista del usuario de la lengua: es su perspectiva la que permite a los sintagmas que involucran eventualidad participar de procesos que involucran una cierta duración, tienen delimitación, y son procesos complejos. Por ejemplo, en 15 ('la negrura del fuel lanzado a sus costas tras el accidente del Prestige les provoca una valoración del futuro del mismo color.'), quien relata el accidente del Prestige se concentra en el estado de negrura que tenía el combustible y lo concibe como un estado concreto que puede causar/provocar angustia en las personas. No parece estar denotando la propiedad de ser negro el petróleo, y sí el estado de negrura que tenía aquel petróleo en aquel momento determinado. Lo mismo pasa en el caso en el que se relata la desaparición de la redondez del rostro. Ella parece estar concebida como un estado que tenía el rostro de aquella persona que, con el tiempo, desapareció. Parece claro, entonces, que en esos casos prima la lectura de eventualidad propuesta por Alexiadou (2013) y no la de subtipo (subespecie) de color/forma determinada. Esto es esperable pues, como mencionamos, las lecturas de proceso no deberían tener un sujeto que denotase alguna cosa abstracta, y sí un sujeto que denotase alguna cosa concreta, en estos casos, eventualidades (más bien, estados). Podemos, por lo tanto, establecer que estas nominalizaciones tienen una lectura concreta cuando son sujeto de realizaciones.

Nos enfocamos, a continuación, en las oraciones que pueden ser clasificadas como estados. Queremos ver si, en estos casos, se presenta solo la lectura abstracta o si se presenta, también, la lectura concreta. Esperamos que sea posible establecer solo la lectura abstracta, pero, si la concreta existe, esperamos poder definir qué estructura poseen los sintagmas que les permite relacionarse con lo abstracto o con lo concreto. Las oraciones que pueden ser clasificadas como estados son claramente 13, 14, 18, 20, 21, 22 y 24. Las que pueden ser clasificadas como estados menos claramente son la 17 y la 23. La 17 ('El azul del cielo y el rojo de los atardeceres se deben a la dispersión de la luz solar') parece involucrar causalidad, efecto y consecuencia. Esto nos permite interpretarla como proceso. Sin embargo, puede ser entendida como un estado, 
lectura ayudada por el tiempo presente de la oración y por la estructura aparentemente genérica. Esta ambigüedad es normal en los nombres, pero debemos determinar cuándo se comporta de una manera y cuándo de otra. La oración 23 ('el verde de las primeras hojas anuncia el triunfo de la vida') parece, a primera vista, poder ser clasificada como realización, debido al verbo 'anunciar'. Ahora, el presente del verbo parece permitir una lectura estativa, que es ayudada por el uso algo metafórico de la oración como un todo. Entonces, la interpretación de estas dos oraciones no es muy clara, pero ambas presentan una ambigüedad interpretativa que no es rara y que nos permite analizarlas como estados en alguna de sus lecturas.

Las oraciones estativas analizadas pueden tener funcionando como sujeto, por un lado, nombres no sufijados de color $(14,17,18,20,21,22$ y 23) y, por otro lado, nominalizaciones deadjetivales (13 y 24). Separamos las oraciones que contienen un tipo de nombre de las que contienen el otro. Consideramos que, como el proceso de formación de ellos es diferente, pueden presentar un comportamiento sintácticosemántico diferente. Analizamos, en primer lugar, las oraciones que contienen nombres no sufijados y, en segundo lugar, las oraciones que contienen nombres sufijados.

Las oraciones 14, 18, 20, 21 y 22 son estativas y tienen como sujeto nombres de color no sufijados. Esos nombres deben ser leídos como estativos y relacionados con estados (aquí considerados subtipos de eventos) concretos. Los nombres de color que forman parte de los sintagmas que funcionan como sujeto tienen género masculino, número singular o plural, imponen la concordancia tanto en el sintagma como en la oración, y pueden ser modificados por adjetivos. La oración 14 ('Los grises del fondo, el azul del cuello y el negro de la sotana, demuestran una voluntad de estilo que se inscribe en el concepto de la pintura-pintura') presenta el nombre 'gris' en plural, muestra la concordancia interna del sintagma (el artículo definido se presenta también en plural) y, también, la concordancia del sujeto complejo con el predicado verbal en la tercera persona del plural. La oración 20 ('el rojo brillante de la cubierta se impone sobre el resto del espacio') puede ser utilizada como evidencia de que esos nombres pueden ser modificados por adjetivos. En este caso en particular, el adjetivo que modifica el nombre es 'brillante'. Es posible, entonces, establecer que, en los casos analizados de oraciones predicativas estativas, los nombres de color no sufijados prefieren una lectura concreta, de estado. Solo por eso, pueden alegrar el ambiente, ser causados por otra cosa, imponerse o servir de marco de referencia para llegar a algún lugar. Por lo tanto, mismo si hay una asociación clara con el tono del color, son leídas como concretas y no como abstractas. Los otros dos casos, que presentan una lectura que no puede ser definida tan claramente como estativa, solo sirven para reforzar el argumento aquí dado: si hubo dificultades para definir si las oraciones 17 ('El azul del cielo y el rojo de los atardeceres se deben a la dispersión de la luz solar ') y 23 ('el verde de las primeras hojas anuncia el triunfo de la vida') eran estados o realizaciones, 
fue solo porque aquellos nombres dentro del sintagma tienen una lectura concreta. Por lo tanto, ninguno de los nombres de color no sufijados aquí usados denotan lo abstracto, ellos denotan la concreto. Teniendo esto en consideración, el análisis de McNally y de Swart (2011) sobre los nombres de color no sufijados no puede ser trasladada al español, pues se prefiere la lectura concreta, y no la lectura abstracta (al menos cuando aparecen complementados).

Continuamos con las oraciones con nombres de color sufijados. Son menos frecuentes, y la lectura estativa parece permitir una lectura concreta en 24 , y una no concreta en 13 (que puede ser extendida a las copulativas 8 y 9). La lectura concreta de 24 ('sobresale el esplendor de los tomates o el verdor de los pimientos'), de 'verdor', es observada a partir de la posibilidad de que el verdor de los pimientos sobresalga en una escena. Hay, por lo tanto, una lectura concreta involucrada. Para que la lectura abstracta exista, las nominalizaciones deben aparecer en el contexto sintácticosemántico propicio para tal lectura, como en 8 ('La altura de este cerro es de $4872 \mathrm{~m}$ sobre el nivel del mar'), 9 ('quizá sea el verdor de los bosques mediterráneos lo más especial y exclusivo') y 13 ('la blancura de las casas del centro del país constituía una especie de legado colonial'). No pueden variar en número y deben presentarse, aparentemente, en singular como en 13. La variación de número implica una variación en el significado del sujeto, la transformación del nombre al plural transforma la lectura no concreta en una lectura concreta, como puede observarse en 13':

(13') las blancuras de las casas del centro del país constituían una especie de legado colonial $^{11}$

En 13', podemos imaginar que 'las blancuras' son manchas en las casas o alguna cosa que es vista como concreta. No se lee la nominalización como algo que pueda llegar a ser abstracto, como un tipo o una clase. Las nominalizaciones que analizamos no aparecen modificadas por ningún adjetivo, pero es posible encontrar casos como:

(26) La blancura total de este cuadro representa, para el movimiento suprematista, la última expresión de la pureza a través del arte.

La modificación parece forzar, nuevamente, una lectura concreta del sintagma. Para hablar de la blancura total, parece ser que el color debe ser percibido, y, por lo tanto, habría una lectura concreta. Que sea representativa de la 'expresión de la pureza' parece confirmar la idea de que hay sí una lectura concreta, de una realización, que permite una valoración de aquello que es percibido.

Podemos defender, entonces, a partir de esos datos -aun siendo ellos cuantitativamente irrelevantes- que, en este tipo de construcciones, las nominalizaciones sufijadas no presentan una lectura concreta. Podemos defender, también, que si esas nominalizaciones apareciesen en plural o modificadas por adjetivos, la lectura cambiaría y pasaría a ser concreta. Por lo tanto, para que tengan la 
lectura no concreta, deben mantenerse en una forma aparentemente singular, no pueden ser modificadas ni pluralizadas. Esto nos lleva a plantearnos que la sintaxis es la que establece si la lectura es concreta o si la lectura es abstracta y no el contenido léxico de la nominalización.

Debemos, entonces, analizar cuál es la estructura que permite que sean leídas como no concretas y qué las diferencia de aquellas que tienen una lectura concreta. Tenemos que ver si la estructura sintáctica de los sintagmas que forman nos permite relacionarlas con tipos o si nos permite relacionarlas con individuos concretos. Para tener esta última lectura, parece ser necesario que sean contables, puedan variar en número y ser modificados sin cambiar de lectura (Zamparelli, 2017). Esto parece confirmar el argumento de que la estructura de los sintagmas debe ser lo que cambia para que estos nombres abstractos pasen de tener una lectura abstracta a una concreta y viceversa, y no parece ser viable establecer que sea el predicado el que transforma la lectura de concreto a abstracto.

Podemos, entonces, preguntarnos si es posible analizar nuestros sintagmas, que parecen denotar entidades abstractas como los genéricos en posición de sujeto, como en 'El león es un mamífero' o 'Gold is a precious metal' (Krifka, Pelletier, Carlson, ter Meulen, Link \& Chierchia, 1995). Se suele trabajar con nombres comunes (con un significado concreto de base), al hablar de los genéricos que se refieren a tipos/especies. No es común encontrar investigaciones sobre genéricos donde se analicen sintagmas formados a partir de nominalizaciones deadjetivales. ${ }^{12}$ Aún así, tratar sintagmas no complementados como 'la blancura' y 'la altura' como genéricos parece ser bastante coherente y compatible con el análisis de McNally y DeSwart (2011). El mismo Carlson (1977) acepta que los nombres abstractos podrían ser tratados como genéricos, pues parecen designar tipos/especies. Sin embargo parece bastante problemático extender este análisis para los casos en los que la nominalización aparece complementada (como en nuestras oraciones), pues el sintagma parece denotar la ejemplificación de aquella propiedad en objetos. ${ }^{13}$

Los tests de genericidad (Krifka et al., 1995) normales no son aplicables a nuestras nominalizaciones, pues están pensados para tipos/especies relacionados con objetos concretos y no para nombres relacionados con lo abstracto. Entonces, no es posible combinar ninguno de nuestros sintagmas con predicados como 'morir' o 'extinguir', ni hacer que nuestros sintagmas sean el objeto de verbos como 'inventar' o 'exterminar'. Tampoco pueden ser caracterizados como tipos/especies bien establecidos (Krifka et al., 1995). Aunque, como Dayal (2004: 425) argumenta, es posible tener genéricos sobre clases que no estén bien establecidas, pues dicha definición se debe más a un efecto pragmático "(...) rather than an inherent restriction on singular kind formation". Es posible afirmar, entonces, que las interpretaciones genéricas son heterogéneas, ya que -como Laca (1999: 902) establece- están definidas solamente "por una propiedad negativa, precisamente, la de no referirse a objetos (o grupos de objetos o porciones 
de materia) individuales". Así, es posible defender que hay una interpretación genérica posible de nuestros sintagmas, mismo cuando no serían genéricos prototipo.

Podemos determinar, por lo tanto, que las nominalizaciones aquí analizadas tienen una lectura abstracta cuando pueden ser tratadas como genéricos. Esto nos lleva a analizar la estructura de los genéricos, para demostrar que la diferencia entre la lectura abstracta y la concreta puede deberse a una variación de la estructura y no a una variación léxica del ítem. Como trabajamos con el español, hacemos nuestro análisis a partir de los de Borik y Espinal $(2012$, 2015). Las autoras proponen que, en el caso de sintagmas definidos genéricos singulares del español, como los nuestros, es posible argumentar que esos sintagmas no presentan la información de número. Según las autoras, los nombres se unen al determinante (el artículo definido) - interpretado como un operador iota que le permite al sintagma denotar tipos/especies - sin mediación de número. Ellas afirman que los definidos genéricos singulares presentan la estructura [sDD [SN N]], y no la estructura canónica de los sintagmas definidos singulares, que es [SD D [ sNum Num [ SN N]]] (Borik \& Espinal, 2012: 128). ${ }^{14} \mathrm{El}$ definido genérico singular no tendría una interpretación individual de objeto, pues no está unido al número singular que permitiría interpretar el sintagma como refiriéndose a un determinado objeto (como en el caso de 'la tele está prendida', donde el sintagma canónico permite referir al objeto TV). Según las autoras, los genéricos definidos singulares se refieren a los tipos/especies en sí mismos, como entidades enteras y sin estructura interna, y no a la suma de los individuos que pertenecen al tipo o la clase, lo que nos permitiría extender el análisis a este tipo de nominalizaciones complementadas.

Algunas modificaciones deben ser hechas a la propuesta de Borik y Espinal (2012, 2015). En primer lugar, queremos defender que, como el número no está presente en el SD, no pueden tener una lectura concreta. Para que tengan esa lectura, el número debe estar presente y los sintagmas deben tener la estructura canónica no marcada [SD D [sNum Num [sN N]]]. En segundo lugar, queremos defender que esos sintagmas no son referenciales, porque no tienen la unicidad asociada a ellos. ${ }^{15}$ En tercer lugar, defendemos que esos sintagmas sí denotan (en el sentido usado por Lyons, 1977) y que esa denotación puede estar asociada a tipos/especies. Cuando usamos un sintagma como 'la blancura de las casas', podemos asociarlo a alguna cosa abstracta relacionada con un tipo determinado.

Finalmente, podemos defender que, cuando hablamos de genéricos identificando tipos/especies, ellos pueden ser asociados a distintas entidades abstractas pues no hay una definición precisa de qué sería un tipo/especie. Podemos encontrar afirmaciones como "(...) with kinds we abstract away from particular objects (...)" (Krifka et al, 1995: 4), defensas como "We will assume throughout that what we call kind-referring noun phrases actually do refer to kinds, which are modeled as special types of individuals" (Krifka et al., 1995: 6), o verlos definidos simplemente como "(...) entities of an argumental type (i.e., of type e)" 
(Chierchia, 1998: 344). El concepto de tipo/especie asociado al individuo usado en la tesis de Carlson (1977) parece ser totalmente compatible con considerar cualquier lectura abstracta como equivalente a los tipos/especies: “... kinds, though, may function is [sic] such a way as to be in many places at a given time" (Carlson, 1977: 69). Podemos considerar que, como los tipos/especies pueden estar en muchos lugares en un determinado tiempo, son términos dados para denotar entidades abstractas y deben ser asociados a las lecturas abstractas de nuestras nominalizaciones. Por lo tanto, estas nominalizaciones en los respectivos SD tienen una lectura abstracta cuando el número no está presente, y una lectura concreta (que puede ser de eventualidad o de cosa) cuando forman un SD canónico no marcado.

\section{CONCLUSIONES}

Hemos analizado las lecturas abstractas y concretas que tienen las nominalizaciones a partir de adjetivos intersectivos no evaluativos. Hemos determinado que las diferentes interpretaciones se deben a una diferencia estructural y no a una variación de significado en el ítem léxico. Esto nos permite tener un léxico más reducido cuyo significado de base sea uno (con una única entrada en el léxico), y explicar la diferencia entre una lectura abstracta y una concreta (y viceversa) a partir de la estructura del SD que el sustantivo forma. La lectura concreta de estos nombres (y de los nombres en general) se debería a la formación de un SD canónico, mientras que la abstracta se debería a la formación de un SD no canónico sin la información de número. Esta idea parece presentar una solución parsimoniosa respecto a estas diferencias interpretativas, lo que parece hacerlo un enfoque prometedor.

\section{REFERENCIAS BIBLIOGRÁFICAS}

Alexiadou, A. (2011). Adjectival nominalizations: qualities and properties. Ponencia presentada en el Workshop on the Syntax and Semantics of Nounhood and Adjectivehood [en línea]. Disponible en: http:/ / filcat.uab.es/clt/Adjectivehood/Presentacions/Adjectival\%20nominal izationsartemis.pdf

Alexiadou, A. (2013). Deriving color adjectival nominalizations. Revista de Estudos Linguísticos da Univerdade do Porto, 8, 143-158.

Arche, J.M. (2016). Aspecto léxico. En J. Gutiérrez-Rexach (Ed.), Enciclopedia de Lingüística Hispánica. Vol. 1 (pp. 405-415). Londres/Nueva York: Routledge.

Baker, M. (2003). Lexical Categories: Verbs, Nouns, and Adjectives. Cambridge/ Nueva York: Cambridge University Press.

Borik, O. \& Espinal, M. T. (2012). On definite kinds. Recherches linguistiques de Vincennes, 41, 123-146 [en línea]. Disponible en: http:// rlv.revues.org/2104. 
Borik, O. \& Espinal, M. T. (2015). Reference to kinds and to other generic expressions in Spanish: Definiteness and number. The Linguistic Review, 32(2), $167-225$.

Carlson, G. (1977). Reference to kinds in English. Tesis doctoral, University of Massachusetts Amherst, Amherst, USA.

Chierchia, G. (1982). Nominalization and Montague grammar: A semantics without types for natural languages. Linguistics and Philosophy, 5, 303-54.

Chierchia, G. (1998). Reference to Kinds across Languages. Natural Language Semantics, 6, 339-405.

Dayal, V. (2004). Number marking and (in)definiteness in kind terms. Linguistics and Philosophy, 27, 393-450.

Den Dikken, M. (2001). Specificational copular sentences and pseudoclefts: A case study. En M. Everaert \& H. van Riemsdijk (Eds.), The Syntax Companion [en línea]. Disponible en: https://www.gc.cuny.edu/CUNY_GC/media/CUNYGraduate-Center/PDF/Programs/Linguistics/Dikken/pseudocl-001.pdf.

Espinal, T. (2013). Bare nominals, bare predicates. Properties and related types. En J. Kabatek \& J. Wall (Eds.), New Perspectives on Bare Noun Phrases In Romance and Beyond (pp. 63-94). Ámsterdam/Philadelphia: John Benjamins.

Fábregas, A. (2011). Concepts, countability and noun classes: On event nouns. Ponencia presentada en JeNom 4, 4èmes Journées d'étude sur les nominalisations, Universidad de Stuttgart, Alemania [en línea]. Disponible en: http://ifla.unistuttgart.de/institut/mitarbeiter/florian/jenom/Antonio-Concepts.pdf.

Hansen, N. \& Chemla, E. (2017). Color adjectives, standards, and thresholds: An experimental investigation. Linguistics and Philosophy, 40(3), 239-78 [en línea]. Disponible en: http://centaur.reading.ac.uk/68143/.

Higgins, F. R. (1979). The pseudo-cleft construction in English. Nueva York: Garland.

Iordăchioaia, G. (2013). The determiner restriction in nominalizations. Ponencia presentada en Languages with and without Articles, Paris [en línea]. Disponible en: http://archive.sfl.cnrs.fr/sites/sfl/IMG/pdf/LSALAA2013Iordachioaia.pdf.

Kennedy, C. \& McNally, L. (2010). Color, context and compositionality. Synthese, 174, 79-98.

Klein, N. M., Gegg-Harrison, W. M., Carlson, G. N. \& Tanenhaus, M. K. (2013). Experimental investigations of weak definite and weak indefinite noun phrases. Cognition, 128, 187-2013. 
Krifka, M., Pelletier, F. J., Carlson, G., ter Meulen, A., Link, G. \& Chierchia, G. (1995). Genericity: An introduction. En G. Carlson \& F. Pelletier (Eds.), The Generic Book (pp. 1-124). Chicago: University of Chicago Press.

Laca, B. (1999). Presencia y ausencia de determinante. En I. Bosque \& V. Demonte (Dirs.), Gramática Descriptiva de la Lengua Española (pp. 891-298) Madrid: Espasa.

Leborans, M. F. (1991). Aspectos semánticos y sintácticos de las oraciones identificativas «inversas». DICEND A. Cuadernos de Filología Hispánica, 10, 7310.

Leborans, M. F. (1999). La predicación: Las oraciones copulativas. En I. Bosque \& V. Demonte (Dirs.), Gramática Descriptiva de la Lengua Española (pp. 2357-2460). Madrid: Espasa.

Lyons, J. (1977). Semantics. Volume I. Cambridge: Cambridge University Press.

Martin, F. (2010). Stage-level and individual level readings of quality nouns: $A$ study in aspectual morpho-semantics. Ponencia presentada en Going Romance 23, Leiden.

McNally, L. \& de Swart, H. (2011). Inflection and derivation: How adjectives and nouns refer to abstract objects. Proceedings of the 18th Amsterdam Colloquium, 425-434.

McNally, L. \& de Swart, H. (2015). Reference to and via properties: The view from Dutch. Linguistics and Philosophy, 38, 315-362.

Morimoto, Y. \& Pavón Lucero, M. V. (2006). Los verbos pseudo-copulativos modales del español [en línea]. Disponible en: http://orff.uc3m.es/bitstream/handle/10016/13865/modales_pavon_VIICL G_2006.pdf?sequence $=1$

Polakof, A. C. (2017). Eventos, fatos e estados de coisas: Da ontologia lógica à linguagem em uso. Tesis doctoral, Pontificia Universidade Católica do Rio de Janeiro, Brasil [en línea]. Disponible en: https://www.maxwell.vrac.puc-rio.br/31527/31527.pdf

Polakof, A. C. (en prensa). Hechos, eventos y la referencia. Análisis Filosófico.

Real Academia Española. (2016). CORPES. DATOS GENERALES [en línea]. Disponible en: http://web.frl.es/CORPES/org/publico/pages/ayuda/informacion.view

Real Academia Española (2018). Diccionario de la Lengua Española [en línea]. Disponible en: http:/ /dle.rae.es/?w=diccionario 
Real Academia Española \& Asociación de Academias de la Lengua Española (2010). Nueva Gramática de la Lengua Española. Manual. Madrid: Espasa.

Roy, I. (2010). Deadjectival nominalizations and the structure of the adjective. En A. Alexiadou \& M. Rathert (Eds.), The Syntax of Nominalizations Across Languages and Frameworks (pp. 129-58). Berlín: Walter de Gruyter.

Vendler, Z. (1967). Linguistics in Philosophy. Ithaca/Londres: Cornell University Press.

Villalba, X. (2013). Eventualitites under deadjectival nominalizations [en línea]. Disponible en: $\quad$ http://semanticsarchive.net/Archive/Tg2NzQ4Z/eventualitiesnominalizations.pdf

Zamparelli, R. (2017/preprint). Countability shifts and Abstract Nouns [en línea]. Disponible en: https://www.researchgate.net/publication/321609637

\section{NOTAS}

${ }^{1}$ Estas características de las nominalizaciones deadjetivales también fueron notadas por Villalba (2013) para casos como 'belleza', a partir de adjetivos evaluativos.

2 Solo analizamos dos formas que no son de color, porque hay pocos adjetivos que obedecen las restricciones propuestas que no sean de color.

${ }^{3}$ Entendemos, siguiendo a Alexiadou (2013), que la forma no sufijada se forma a partir de la nominalización de un adjetivo. Por ese motivo, hablamos de nominalización también en el caso de los nombres/términos de color.

${ }^{4}$ Este subcorpus puede verse en Polakof (2017).

${ }^{5} \mathrm{El}$ nombre 'verdura' no puede formarse con el significado de color, porque está bloqueado por la existencia del nombre 'verdura' con el significado de 'hortaliza'.

' Si el sentido intersectivo del adjetivo 'alto' no estuviese en el diccionario, otra explicación debería ser dada. En ese caso, deberíamos explicar cómo la gradabilidad del adjetivo 'alto’ se pierde al formar la nominalización, lo que está relacionado a los opuestos o antónimos gradables (Lyons, 1977).

${ }^{7}$ El problema de la clasificación de las copulativas es muy complejo y ha sido analizado, en lingüística generativa, a partir del trabajo de Higgins (1979). Sin embargo, como Den Dikken (2001) menciona, el problema es mucho más complejo. Creemos que, para el objetivo de nuestra investigación, esta distinción tradicional y simple es más que suficiente.

8 Puede ser considerado no referencial, pues el artículo neutro está determinado por defecto en las construcciones que no son plenamente nominales en español (Iordăchioaia, 2013). 
9 Las oraciones copulativas también podían haber sido analizadas según el aspecto. Sin embargo, presentan características propias y el análisis se ve enriquecido con la separación entre copulativas y predicativas.

${ }^{10}$ Obviamente, Vendler está lejos de ser el único en trabajar con esa noción, pero para nuestros objetivos su clasificación es suficiente.

${ }^{11}$ En el CORPES, 'blancuras' presenta dos registros en posición de objeto y en sintagmas sin determinante, como puede observarse en: '(...) levantaron azules difusos, blancuras calcáreas y pequeñas dunas'.

12 Chierchia (1998) tiene una sección que habla de adjetivos nominalizados como 'the poor', que son construcciones humanas que no analizamos. Zamparelli (2017) tiene un trabajo más enfocado en los nombres abstractos, donde analiza su relación con los términos de masa, pero no se enfoca en este tipo de nominalizaciones.

${ }^{13}$ La interpretación genérica se ve ayudada cuando el complemento está en plural (como en 9 y 13) y se ve un poco más perjudicada cuando el complemento está en singular (como en 8).

${ }^{14}$ La propuesta de Borik y Espinal (2012, 2015) no está libre de problemas. Particularmente, el tratamiento no unificado de los genéricos singulares y plurales deja un poco que desear. Sin embargo, para los objetivos de este artículo es suficiente.

${ }^{15}$ Esta idea va contra la propuesta de Baker (2003), que establece que los nombres tienen un índice referencial y que, por eso, forman estructuras referenciales. Esta propuesta es problemática. Espinal (2013) muestra, por su lado, que hay nominales escuetos que no presentan un índice referencial y que la propuesta de Baker no puede ser generalizada. 